\title{
Üçlü Ters Parabolik Kuantum Bariyer Çift Kuyu Potansiyelinde Enerji Seviyeleri ile Elektron Geçişinin Yapı Parametreleri ve Yoğun Lazer Alanına Bağlılığının İncelenmesi
}

\author{
Investigation of Relation of Electron Transmission and Energy State to Structure Parameters \\ and The Intense Laser Field in The Triple Inverse Parabolic Quantum Barrier Double Well \\ Potential
}

Mehmet BATI ${ }^{1}$

${ }^{1}$ Recep Tayyip Erdoğan Üniversitesi Fizik Bölümü, 53100, RİZE

$\ddot{O} z$

Bu çalışmanın amacı, yoğun lazer alanı altında GaAs/AlGaAs lardan oluşan üçlü ters parabolik kuantum bariyer çift kuyu potansiyelinde elektronik iletimi ve enerji seviyelerinin bariyer genişlikleri, lazer alanı giydirme parametresine bağlılığını araştırmaktır. Çalışmada Denge-dışı Green fonksiyonları yöntemi kullanılarak iletim olasılıkları ve rezonans enerji seviyeleri tespit edilmiştir. Lazer alanının ve yapı parametrelerinin rezonans tünellemeyi oldukça etkilediği, enerji seviyelerinin yerlerinin kontrolünün bu parametrelerle yapılabildiği görülmüştür. Lazer alanının artmasıyla enerji seviyelerinin daha yüksek enerjilerde ortaya çıktığı görülmüştür. Yapının rezonans tünelleme özelliğinin kontrolü işlevsel nano-aygıt yapımında oldukça önem arz etmektedir.

Anahtar Kelimeler: Denge-Dışı Green Fonksiyonu, Yoğun Lazer Alanı, Rezonans Tünelleme, Çift Kuyu Potansiyeli, Ters Parabolik Bariyer.

\begin{abstract}
The aim of this study is to investigate the dependence of the transmission properties and energy state of the triple inverse parabolic quantum barrier double well potential structure made up of GaAs/AlGaAs under the intense laser field with the well and barrier widths and the laser field dressing parameter. In our work, In the study, transmission probabilities and resonance energy levels were determined using the Non-Equilibrium Green functions method. It has been observed that laser field and structure parameters affect resonance tunneling and control of the location of energy levels can be done with these parameters. It has been observed that energy levels emerge at higher energies as the laser field increases. The control of the resonance tunneling feature of the structure is very important in the production of functional nano-devices.

Keywords: Non equilibrium Green function, Intense Laser Field, Resonant Tunneling, Double Well Potential, Inverse Parabolic Barrier.

\section{GİRIŞ}

Kuantum yapıların iletim özellikleri özellikle rezonans tünelleme olayı yeni fonksiyonel nano cihazların üretimi nedeniyle büyük ilgi görmüştür [1-4]. Örneğin, rezonans tünel diyotları (RTD'ler) ve rezonans tünelleme transistörleri (RTT'ler) tera hertz görüntüleme sistemi [5], yüksek hızlı anahtarlama ve yüksek hızlı mantık uygulamaları [6] olarak araştırılmıştır. Nano-fabrikasyon teknolojisindeki son gelişmeler, çok çeşitli potansiyel şekilleri olan rezonans tünelleme yapılarının üretilmesini mümkün kılmıştır. Düşük-boyutlu yarı iletken yapı üretimindeki son gelişmeler nano-yapılara dayalı elektronik cihazların karakteristiklerinin belirlenmesinde önem arz eden elektron taşınımının kontrol edilebilirliği için yeni olanaklar sağlamaktadır. Düşük-boyutlu yarıiletken yapılarda boyut azalmasıyla baskın hale gelen kuantum mekaniksel etkiler külçe yapılara göre farklı fiziksel olayların görülmesine neden olmaktadır. Son yıllarda, farklı potansiyel profillerinde elektronik iletim incelenmiştir. [7-10]. Birçok çalışma tek kuantum-kuyu ve çift bariyer rezonans tünelleme yapıları üzerine odaklanmıştır [11-17] ancak rezonans tünelleme olayı üçlü bariyer rezonans tünelleme yapılarında da tanımlanmıştır. Potansiyel uygulanabilirlik (alan etkili transistörler, kuantum kuyuları, lazerler, elektro-optik modülatörler ve kuantum kuyu kızılötesi foto detektörleri) nedeniyle, çift kuyu üçlü bariyer rezonans tünel yapıları ilgi çekmiştir [18-21]. Üçlü bariyer RTD' leri ağırlıklı olarak, bellek uygulamalarında yüksek oranda kullanılan çoklu negatif diferansiyel direnç (NDR) bölgelerine sahip cihazın elektrik özelliklerinde çoklu tepe noktaları oluşturmak için kullanılmaktadır [22-24].
\end{abstract}


Yoğun bir lazer alanının düşük boyutlu yarı iletkenlerin fiziksel özellikleri üzerindeki etkileri, yüksek güçlü, uzun dalga boylu, doğrusal polarize lazer kaynaklarının ortaya çıkması nedeniyle dikkate değer bir konudur. $\mathrm{Bu}$ durum lazer alanı ile ilişkili ilginç fiziksel olayların keşfini başlatmıştır [25-34]. Yoğun lazer 1şı̆̆ının madde ile etkileşmesini inceleyen çalışma $\mathrm{THz}$ darbelerinin yönlendirilmesi, odaklanması, yeniden şekillendirilmesi ve teşhisine yönelik mevcut yaklaşımları içermektedir [32]. GaAs- $\mathrm{Ga}_{1-\mathrm{x}} \mathrm{Al} \mathrm{x}_{\mathrm{x}} \mathrm{As}$ kuantum kuyularında farklı hapsedilme potansiyeline sahip kuantum kuyularında bant içi geçişler üzerindeki yoğun lazer alanı etkileri Niculescu ve ark. tarafindan incelenmiştir [33]. Yoğun lazer alanları altındaki asimetrik GaAs/GaAlAs çift kuantum kuyularında elektrik ve manyetik alanların optik özellikler üzerindeki etkileri teorik olarak Yesilgul ve ark. [34] tarafından çalışılmıştır. Saczuk ve arkadaşları, çift bariyer yapılarından elektronların rezonans iletiminin yoğun lazer alanları ile önemli ölçüde değiştirilebildiğini ve kontrol edilebildiğini göstermiştir [35]. Aktaş ve ark. simetrik ve asimetrik çift bariyer yapısında yoğun lazer alanının elektron iletimine etkilerini araştırmışlardır. $\mathrm{Bu}$ çalışmalar, sistemin yoğun bir lazer alanı ile aydınlatıldığında, sistemin iletim özelliklerindeki kayda değer değişikliklerin meydana geldiğini göstermektedir [36].

Yoğun bir lazer alanının farklı yapılar üzerindeki etkilerinin araştırılmasına büyük ilgi gösterilmesine rağmen, yoğun lazer alanı altındaki üçlü ters parabolik bariyer çift kuyu (PBÇK) yapısının elektronik özellikleri şu ana kadar incelenmemiştir. Parabolik bariyerler, gerçek bir bariyerin gerçekçi bir şekilde temsil edilmesinden dolayı fizik ve kimyada özel bir öneme sahiptir. Bu çalışmada, yoğun lazer alanının simetrik PBÇK yapısında bir elektronun geçiş olasılığ ve enerji durumları üzerindeki etkisini inceleyeceğiz. Enerji durumlarını ve iletim katsayısını bulmak için denge dişı Green fonksiyonu (NEGF) yöntemini kullanıyoruz. NEGF yöntemi, açık kuantum sistemlerde taşıyıcı dinamikleri tanımlamak için en etkili ve genel yaklaşımlardan biridir [37].

\section{MATERYAL VE METOT}

$\mathrm{Bu}$ etkin kütle yaklaşımında, yoğun lazer alanının varlığında bir elektron için zamana bağlı Schrödinger denklemi aşağıdaki gibi verilir [15,26].

$\left[\frac{(\vec{P}+e \vec{A})^{2}}{2 m^{*}}+V_{0}(x)\right] \psi(x, t)=i \hbar \frac{\partial}{\partial t} \psi(x, t)$

$m^{*}$ elektron etkin kütlesi, $V_{0}(x)$ sonlu kuşatma potansiyeli, $\vec{A}$ vektör potansiyeli ve $\vec{P}$ momentum operatörüdür.

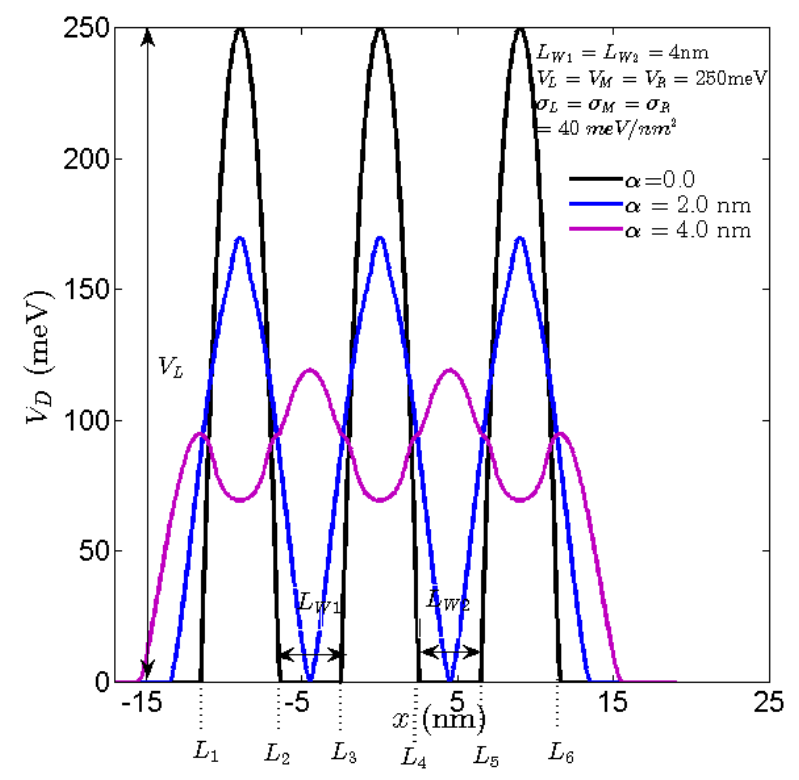

Şekil 1. PBÇK yapısına lazer alanının etkisi

Şekil 1 de verilen PBÇK potansiyelin lazer alanı yokluğundaki fonksiyonel formu

$V_{0}(x)=\left\{\begin{array}{rr}-\sigma_{L}\left(x-x_{m L}\right)^{2}+V_{L}, & L_{1} \leq x \leq L_{2}, \\ -\sigma_{M}\left(x-x_{m M}\right)^{2}+V_{M}, & L_{3} \leq x \leq L_{4}, \\ -\sigma_{R}\left(x-x_{m R}\right)^{2}+V_{R}, & L_{5} \leq x \leq L_{6}, \\ 0, & \text { Diğer yerlerder. }\end{array}\right.$

Şeklindedir [38]. Burada $\sigma_{L}=2 V_{L} /\left(L_{1}-x_{m L}\right)^{2}+$ $\left(L_{2}-x_{m L}\right)^{2}, \quad \sigma_{M}=2 V_{M} /\left(L_{3}-x_{m M}\right)^{2}+\left(L_{4}-\right.$ $\left.x_{m M}\right)^{2}$ ve $\sigma_{R}=2 V_{R} /\left(L_{5}-x_{m R}\right)^{2}+\left(L_{6}-x_{m R}\right)^{2}$ dir. $V_{L}, V_{M}$ ve $V_{R}$, sirasiyla sol, orta ve sağ bariyer yüksekliğidir. Şekil 1 de, $L_{i}$ (i: $1 \ldots 6$ ), yapıyı oluşturan her bölgenin konumunu belirtirken, bariyer maksimumları $x_{m L}=(L 1+L 2) / 2, \quad x_{m M}=(L 3+$ $L 4) / 2$ ve $x_{m R}=(L 5+L 6) / 2$ konumlarındadir. Sol kuyu genişliği $L_{W 1}=\left|\mathrm{L}_{3}-\mathrm{L}_{2}\right|$, ve sağ kuyu genişliği $L_{W 2}=\left|\mathrm{L}_{5}-\mathrm{L}_{4}\right|$ olmak üzere çalışma boyunca simetrik yapı incelenmiştir $\left(L_{W 1}=L_{W 2}=L_{W}\right.$ ve $\left.V_{L}=V_{M}=V_{R}=V=250 \mathrm{meV}\right)$.

Sisteme uygulanan, monokromatik lineer polarize rezonant olmayan yüksek-frekanslı yoğun lazer alanı yarı-klasik olarak pertürbatif olmayan çerçevede ve $\mathrm{A}(\mathrm{x}, \mathrm{t}) \approx \mathrm{A}(\mathrm{t})$ dipol yaklaşımı kullanılarak ele alınmıştır. Sistemi tanımlayan zamana-bağlı Schrödinger denklemi [26,40];

$\left[-\frac{\hbar^{2}}{2 m^{*}} \frac{d^{2}}{d x^{2}}+V(x+\vec{\alpha}(t))\right] \Phi(x, t)=i \hbar \frac{\partial}{\partial t} \Phi(x, t)$

Lazer alan radyasyonu tarafindan oluşturulan vektör potansiyeli $\vec{A}(t)=A_{0} \sin (\omega t) \hat{i}$. Lazer alanının PBÇK üzerindeki etkisi altında hareket eden bir elektron için zamana bağlı Schrödinger denklemi, KramersHenneberger üniter dönüşümü uygulanarak dönüştürülmüştür [26,31]. $\mathrm{Bu}$ eşitlikte, $\vec{\alpha}(\mathrm{t})=$ $\alpha \cos (\omega t) \hat{\imath}$ parçacığın polarizasyon yönü boyunca salınım merkezinden olan uzaklığını betimleyen 
vektördür. Burada $\quad \alpha=\frac{\mathrm{eA}_{0}}{\mathrm{~m}^{*} \omega} \quad$ lazer giydirme parametresidir.

Sistemi tanımlayan zamana-bağlı Schrödinger denklemi Krammers-Henneberger dönüşümü ve Fourier-Floquet seri açılımı kullanılarak (yüksek frekans limitinde), lazer giydirilmiş (laser-dressed) potansiyeli içeren zamandan-bağımsız formda [26-40],

$\left[-\frac{\hbar^{2}}{2 m^{*}} \frac{d^{2}}{d x^{2}}+V_{D}(x+\alpha)\right] \Phi(x)=E \Phi(x)$

elde edilir. Burada $\mathrm{V}_{\mathrm{D}}(\mathrm{x}+\alpha)$ lazer giydirilmiş potansiyeldir ve

$V_{D}(x+\alpha)=\frac{\omega}{2 \pi} \int_{0}^{2 \pi / \omega} V(x+\alpha(t)) d t$

ile verilir. Sayısal kolaylık açısından Schrödinger eşitliğini etkin Bohr yarıçapı $\left(\mathrm{a}_{0}^{*}\right)$ ve Hartree enerjisi $\left(\mathrm{E}_{\mathrm{H}}^{*}\right)$ ölçeklendirirsek

$-\frac{1}{2} \frac{d^{2}}{d \tilde{x}^{2}}+\widetilde{\mathrm{V}}_{D}(\tilde{\mathrm{x}}, \tilde{\alpha}) \Phi=\widetilde{\mathrm{E}} \Phi$

Elde edilir. Buradaki sembol boyutsuz parametreler (ölçeklendirilmiş parametreler) için kullanılmıştır. Eşitlik (6) ya sonlu farklar metodu uygulanırsa [37]

$-\tilde{t} \Phi_{n-1}+\left(2 \tilde{t}+\tilde{V}_{D_{n}}\right)-\tilde{t} \Phi_{n+1}=E \Phi_{n}$

burada $\tilde{\mathrm{t}}=\frac{1}{2 \Delta \tilde{\mathrm{x}}^{2}} \quad$ sıçrama parametresidir ve en yakın komşu bölgeler arasındaki etkileşimi temsil etmektedir. $\widetilde{\mathrm{V}}_{\mathrm{D}_{\mathrm{n}}}=\widetilde{\mathrm{V}}_{\mathrm{D}}\left(\widetilde{\mathrm{x}}_{\mathrm{n}}\right)$ olarak kısaltılmıştır. Öz enerji terimleri eklenerek Eşitlik (7) matris formda Eşitlik (8) şeklinde tanımlanır [37].

$\left[\widetilde{\mathrm{E} I}-\widetilde{\mathrm{H}}-\tilde{\Sigma}_{\mathrm{L}}-\widetilde{\Sigma}_{\mathrm{R}}\right]\{\Phi\}=\{\widetilde{\mathrm{S}}\}$

Eşitlik (8) de $[\widetilde{H}]$ Hamiltonyen matrisi, [I] birim matris, $\{\Phi\}$ dalga fonksiyonu vektörü ve $\{\widetilde{S}\}$ saçılma terimi vektörüdür. $\widetilde{\Sigma}_{\mathrm{L}}$ ve $\widetilde{\Sigma}_{\mathrm{R}}$ sırasıyla sağ ve sol kontağın öz enerji terimleridir. Böylece

$[\widetilde{H}]=\left(\begin{array}{ccccccc}2 \tilde{\mathfrak{t}}+\widetilde{V}_{\mathrm{D}_{1}} & -\tilde{\mathrm{t}} & 0 & & \cdots & 0 & 0 \\ -\tilde{\mathrm{t}} & 2 \tilde{\mathrm{t}}+\widetilde{\mathrm{V}}_{\mathrm{D}_{2}} & -\tilde{\mathrm{t}} & & \cdots & 0 & 0 \\ 0 & -\tilde{\mathrm{t}} & 2 \tilde{\mathrm{t}}+\widetilde{\mathrm{V}}_{\mathrm{D}_{3}} & & \ddots & \vdots & \vdots \\ 0 & 0 & \ddots & & -\tilde{\mathrm{t}} & 0 & \\ \vdots & \vdots & \ddots & -\tilde{\mathrm{t}} & 2 \tilde{\mathrm{t}}+\widetilde{\mathrm{V}}_{\mathrm{D}_{N-1}} & -\tilde{\mathrm{t}} \\ 0 & \cdots & 0 & 0 & -\tilde{\mathrm{t}} & 2 \tilde{\mathrm{t}}+\widetilde{\mathrm{V}}_{\mathrm{D}_{N}}\end{array}\right)$

Formunda yazılabilir. $\tilde{\mathrm{k}}_{\mathrm{L}}$ ve $\tilde{\mathrm{k}}_{\mathrm{R}}$ sağ ve sol kontak düzlem dalga fonksiyonu dalga vektörleri olmak üzere; öz enerji terimleri $\left[\Sigma_{\mathrm{L}}\right],\left[\Sigma_{\mathrm{R}}\right]$ ve saçılma terimi $\{\mathrm{S}\}$

$$
\begin{aligned}
{\left[\widetilde{\Sigma}_{\mathrm{L}}\right] } & =\left(\begin{array}{ccccc}
-\tilde{t} e^{i \tilde{k}_{L} \Delta \tilde{x}} & 0 & \ldots & 0 \\
& 0 & 0 & & 0 \\
& \vdots & & \ddots & \vdots \\
& 0 & & \cdots & 0
\end{array}\right), \\
{\left[\widetilde{\Sigma}_{\mathrm{R}}\right] } & =\left(\begin{array}{cccc}
0 & \cdots & & 0 \\
\vdots & \ddots & & \vdots \\
0 & \ldots & 0 & 0 \\
0 & \cdots & 0 & -\tilde{t} e^{i \tilde{k}_{R} \Delta \tilde{x}}
\end{array}\right),
\end{aligned}
$$

$\{\tilde{\mathrm{S}}\}=\left(\begin{array}{c}-\tilde{t}\left(e^{i \tilde{k}_{L} \Delta \tilde{x}}-e^{-i \tilde{k}_{L} \Delta \tilde{x}}\right. \\ 0 \\ \vdots \\ 0\end{array}\right)$,

olarak elde edilir. Kuantum sisteminin Green fonksiyounu matris formda aşağıdaki gibi tanımlanır [37].

$\left[\mathrm{G}^{\mathrm{r}}\right]=\left[(\widetilde{\mathrm{E}}+\mathrm{i} \lambda) \mathrm{I}-\widetilde{\mathrm{H}}-\widetilde{\Sigma}_{\mathrm{L}}-\widetilde{\Sigma}_{\mathrm{R}}\right]^{-1}$

Burada $\lambda$ sonsuz küçük pozitif bir sayıdır. Geçiş olasılı̆̆ $1(\mathrm{~T})$

$\mathrm{T}=\operatorname{Tr}\left[\tilde{\Gamma}_{\mathrm{L}} \mathrm{G}^{\mathrm{r}} \tilde{\Gamma}_{\mathrm{R}} \mathrm{G}^{\mathrm{r}+}\right]$

ifadesi ile hesaplanır. Burada $\tilde{\Gamma}_{\mathrm{L}}=\mathrm{i}\left[\tilde{\Sigma}_{\mathrm{L}}-\tilde{\Sigma}_{\mathrm{L}}^{+}\right]$ve

$\tilde{\Gamma}_{\mathrm{R}}=\mathrm{i}\left[\tilde{\Sigma}_{\mathrm{R}}-\tilde{\Sigma}_{\mathrm{R}}^{+}\right] \quad$ genişleme fonksiyonlarıdır.

\section{BULGULAR}

Çalışmamızda sistemin etkin kütlesi yap1 boyunca $m^{*}=0,067 m_{e}, m_{e}=9,109410^{-31} \mathrm{~kg}$ serbest elektron kütlesi, dielektirik sabiti $\varepsilon_{r}=12,7$, etkin Bohr yarıçapı $a_{0}^{*}=10,1061 \mathrm{~nm}$, etkin Hartree enerjisi $E_{H}^{*}=11,2193 \mathrm{meV}$ alınmıştır [17]. Bu değerler GaAs temelli yariiletkenlerin parametreleridir. Şekil 2 de iletim olasılığının gelen elektron enerjisi ile değişimi gösterilmektedir. $\mathrm{Bu}$ grafikte kuyu genişlikleri $L_{w}=$ $4.0 \mathrm{~nm}$, bariyer genişlik parametresi $\sigma=40 \mathrm{meV} / \mathrm{nm}^{2}$ seçilmiş olup üç farklı lazer giydirme parametresi ( $\alpha=$ $0.0,2.0$ ve $4.0 \mathrm{~nm}$ ) için çizilmiştir. Şekil 2 de içerisine eklenen küçük şekilde lazer giydirme parametresinin potansiyel profiline etkisi görülmektedir. Sisteme uygulanan yoğun lazer alan şiddeti $\alpha$ ile orantılı olup, $\alpha$ 'nın artması ile PBÇK potansiyel formunum değiştiği gözlemlenmiştir ve buna bağlı olarak rezonans tünelleme geçiş katsayısında oluşan rezonans pikin biraz daha yüksek enerji değerlerinde ortaya çıktığı görülmüştür. Rezonans tünelleme pikleri kuyu içerisinde kalan kuyu enerji seviyesi ile gelen elektronun enerjilerinin aynı olduğu değerlerde oluşmaktadır. Bu enerji seviyelerine rezonans enerji $\left(E_{\text {res }}\right)$ seviyeleri adi verilir. Şekil 2 de elektronun enerjisine (E) bağlı olarak geçiş olasılığı $(\mathrm{T})$ değişimi verilmiştir.

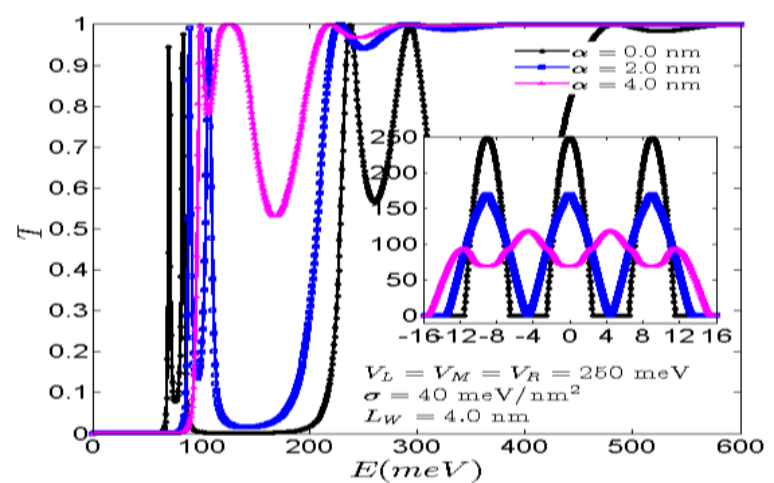

Şekil 2. Geçiş olasılığının elektron enerjisine bağlı değişimi 
meV mertebesinde olan elektronik enerji seviyeleri aralıkları, THz mertebesinde foton frekansına karşılık gelmektedir. Güçlü alan $\mathrm{THz}$ darbeleri kullanılarak potansiyel yapısının değişimi sağlanabilmektedir. Bu durum, manyetik düzen, süperiletken karakter veya ferroelektrik polarizasyon gibi malzeme özelliklerini değiştiren yapı geometrisinin kontrolünü sağlar [3538]. Bu tip nanoyapılar terahertz bant genişliği sinyallerini üretmek ve tespit etmek için kullanılabilen yüksek hızlı anahtarlamaya sahip bazı kuantum cihazları yapılabilmesine olanak sağlamaktadır [2,17].

Rezonans enerji seviyesindeki geçiş olasılığı rezonans pik şiddeti $\left(\mathrm{T}\left(\mathrm{E}_{\text {res }}\right)\right)$ olarak isimlendirilmektedir. Lazer alanı uygulanmamış haldeki potansiyel yüksekliği olan $\mathrm{V}=250 \mathrm{meV}$ değerinden düşük enerji seviyeleri ele alınmıştır.

Tablo 1. PBÇK yapısında rezonans enerji seviyeleri ve bu seviyeler denk gelen elektron enerjilerinde geçiş olasılığ $1\left(\sigma=40 \mathrm{meV} / \mathrm{nm}^{2}, \alpha=0, V_{L}=V_{M}=V_{R}=\right.$ $250 \mathrm{meV}$ )

\begin{tabular}{|c|c|c|c|c|c|c|c|c|}
\hline $\begin{array}{c}L_{\mathrm{W}} \\
(\mathrm{nm})\end{array}$ & $\begin{array}{c}E_{\text {res } 1} \\
\mathrm{meV}\end{array}$ & $\begin{array}{c}E_{\text {res2 } 2} \\
\mathrm{meV}\end{array}$ & $\begin{array}{c}E_{\text {res3 }} \\
\mathrm{meV}\end{array}$ & $\begin{array}{c}E_{\text {res4 }} \\
\mathrm{meV}\end{array}$ & $T_{\text {Eres } 1}$ & $T_{\text {Eres2 } 2}$ & $T_{\text {Eres3 } 3}$ & $T_{\text {Eres4 }}$ \\
\hline 0 & 185 & - & - & - & 0.9998 & - & - & - \\
\hline 2.0 & 109.9 & 138.1 & - & - & 0.997 & 0.9993 & - & - \\
\hline 4.0 & 69.67 & 82.88 & 237.8 & - & 0.9453 & 0.9758 & 0.9998 & - \\
\hline 6.0 & 47.45 & 54.05 & 171.8 & 203 & 0.8548 & 0.8298 & 0.9965 & 1 \\
\hline 8.0 & 34.23 & 37.84 & 128.5 & 146.5 & 0.9753 & 0.3183 & 0.9997 & 1 \\
\hline 10.0 & 25.83 & 28.23 & 99.1 & 109.9 & 0.6764 & 0.972 & 0.9343 & 1 \\
\hline 12.0 & 21.02 & 21.62 & 78.08 & 85.29 & 0.01695 & 0.395 & 0.95 & 1 \\
\hline
\end{tabular}

Tablo 1 de sisteme lazer alanı uygulanmayan $(\alpha=0)$ durumda kuyu genişliğine bağlı olarak enerji seviyelerinin yeri ve bu seviyelere denk enerjide gelen elektronların yapıdan geçme olasılıkları verilmiştir. Genişlik parametresi $\sigma=40 \mathrm{meV} / \mathrm{nm}^{2}$ olarak seçilmiştir. $E_{\text {res } 1}, E_{\text {res } 2}$... geçiş olasılığını maksimum yapan (bazı durumlarda 1 yapan) sirasıyla 1. 2. ...rezonans enerji seviyeleridir. Kuyu genişliği arttıkça rezonans enerji seviyesi daha düşük enerjilere (kırmızıya kayma) doğru kaymakta ve enerji seviyeleri arasındaki fark $(\Delta E)$ azalmaktadır. Kuyu genişliği arttıkça 1. Rezonans enerjisinde geçiş olasılığı azalmaktadır. Bunun nedeni kuyular genişledikçe gelen elektronların kuyularda lokalize olmaya başlamalarıdır. Düşük enerji seviyelerinde bariyerin parabolik yapısından ötürü tünelleme olasılığı azalır.

Tablo 2. PBÇK yapısında rezonans enerji seviyeleri ve bu seviyeler denk gelen elektron enerjilerinde geçiş olasılığ $\left(\sigma=40 \mathrm{meV} / \mathrm{nm}^{2}, \alpha=2 \mathrm{~nm}, V_{L}=V_{M}=\right.$

$$
V_{R}=250 \mathrm{meV} \text { ) }
$$

\begin{tabular}{|l|l|l|l|l|l|l|l|l|}
\hline $\begin{array}{c}L_{W} \\
(\mathrm{~nm})\end{array}$ & $\begin{array}{c}E_{\text {res1 }} \\
\text { meV }\end{array}$ & $\begin{array}{l}E_{\text {res2 } 2} \\
\text { meV }\end{array}$ & $\begin{array}{l}E_{\text {res3 }} \\
\text { meV }\end{array}$ & $\begin{array}{l}E_{\text {res4 }} \\
\text { meV }\end{array}$ & $T_{\text {Eres1 }}$ & $T_{\text {Eres2 }}$ & $T_{\text {Eres3 }}$ & $T_{\text {Eres4 }}$ \\
\hline 0.0 & 192.8 & - & - & - & 0.9999 & - & - & - \\
\hline 2.0 & 129.1 & 165.8 & - & - & 1 & 0.9996 & - & - \\
\hline 4.0 & 88.89 & 106.9 & 228.2 & - & 0.9901 & 0.9871 & 1 & \\
\hline 6.0 & 61.26 & 69.67 & 175.4 & 213.8 & 0.9998 & 0.9204 & 1 & 1 \\
\hline 8.0 & 43.84 & 48.05 & 138.7 & 162.2 & 0.2585 & 0.9999 & 1 & 1 \\
\hline 10.0 & 32.43 & 34.83 & 110.5 & 124.9 & 0.06738 & 0.3292 & 1 & 1 \\
\hline 12.0 & 24.62 & 25.83 & 88.89 & 97.9 & 0.09848 & 0.03311 & 1 & 1 \\
\hline
\end{tabular}


lazer alanının şiddetli olması durumunda kuyu yerleri ve yüksekliklerinin değişmesidir. Yoğun lazer alanı, yapıda büyük yapısal değişikliklere yol açmaktadır.
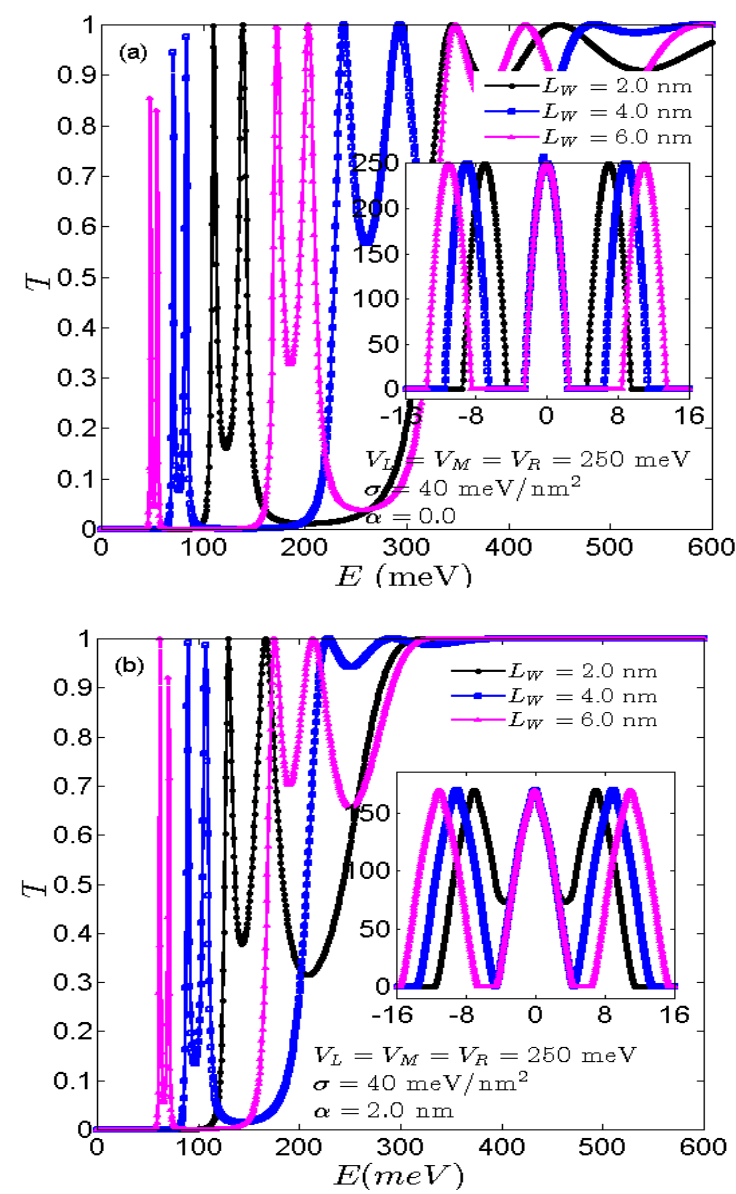

Şekil 3. Geçiş olasılığının farklı kuyu genişlikleri için $\left(\mathrm{L}_{\mathrm{W}}=2.0,4.0,6.0 \mathrm{~nm}\right)$ elektron enerjisine bağl1 değişimi. (a) lazer alanı yokluğunda $(\alpha=0)$, (b) lazer alanı varlığında $(\alpha=2.0 \mathrm{~nm})$ çizilmiştir

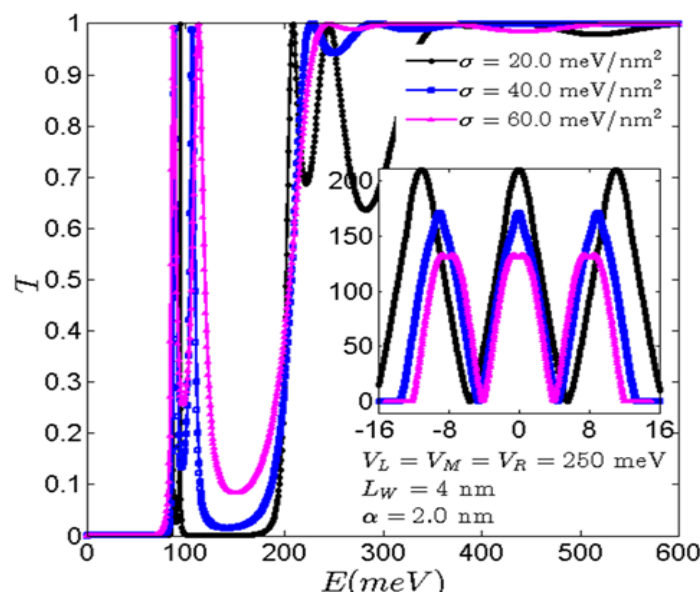

Şekil 4. Geçiş olasılığının farklı genişlik parametreleri için $\left(\sigma=20,40,60 \mathrm{meV} / \mathrm{nm}^{2}\right)$ elektron enerjisine bağlı değişimi.
Tablo 4. PBÇK yapısında rezonans enerji seviyeleri ve bu seviyeler denk gelen elektron enerjilerinde geçiş olasılığ $L_{W}=4 \mathrm{~nm}, \alpha=0 \mathrm{~nm}, V_{L}=V_{M}=V_{R}=$ $250 \mathrm{meV}$

\begin{tabular}{|c|c|c|c|c|c|c|c|c|}
$\begin{array}{c}\boldsymbol{\sigma} \\
\mathrm{meV} / \mathrm{nm}^{2}\end{array}$ & $\begin{array}{c}E_{\text {res1 }} \\
\text { meV }\end{array}$ & $\begin{array}{c}E_{\text {res2 }} \\
\mathrm{meV}\end{array}$ & $\begin{array}{c}E_{\text {res3 }} \\
\mathrm{meV}\end{array}$ & $\begin{array}{c}E_{\text {res4 }} \\
\mathrm{meV}\end{array}$ & $T_{\text {Eres1 }}$ & $T_{\text {Eres2 }}$ & $T_{\text {Eres3 }}$ & $T_{\text {Eres4 }}$ \\
\hline 11.0803 & 63.06 & - & 197 & 212.6 & 0.1674 & - & 1 & 1 \\
\hline 20.4082 & 67.27 & 72.67 & 214.4 & 245.6 & 0.3303 & 0.7901 & 1 & 1 \\
\hline 40.0000 & 69.67 & 82.88 & 237.8 & - & 0.9453 & 0.9758 & 1 & - \\
\hline 51.6529 & 69.67 & 87.09 & 248.6 & - & 0.9513 & 0.984 & 1 & - \\
\hline 62.5000 & 69.67 & 90.09 & - & - & 0.9916 & 0.9979 & - & - \\
\hline 81.6327 & 69.07 & 94.29 & - & - & 0.9941 & 0.9999 & - & - \\
\hline
\end{tabular}

Tablo 5. PBÇK yapısında rezonans enerji seviyeleri ve bu seviyeler denk gelen elektron enerjilerinde geçiş olasılığ $L_{W}=4 \mathrm{~nm}, \alpha=2 \mathrm{~nm}, V_{L}=V_{M}=V_{R}=$ $250 \mathrm{meV}$

\begin{tabular}{|l|l|l|l|l|l|l|l|l|}
$\begin{array}{c}\boldsymbol{\sigma} \\
\text { meV/nm2 }\end{array}$ & $\begin{array}{c}E_{\text {res1 }} \\
\text { meV }\end{array}$ & $\begin{array}{c}E_{\text {res2 } 2} \\
\text { meV }\end{array}$ & $\begin{array}{c}E_{\text {res3 }} \\
\text { meV }\end{array}$ & $\begin{array}{l}E_{\text {res4 }} \\
\text { meV }\end{array}$ & $T_{\text {Eres1 } 1}$ & $T_{\text {Eres2 }}$ & $T_{\text {Eres3 }}$ & $T_{\text {Eres4 }}$ \\
\hline 10 & 78.68 & 80.48 & 193.4 & 210.8 & 0.04263 & 0.865 & 1 & 1 \\
\hline 20.4082 & 86.49 & 94.29 & 209 & 246.2 & 0.9991 & 0.7618 & 1 & 1 \\
\hline 40.0000 & 88.89 & 106.3 & 228.2 & - & 0.9901 & 0.9875 & 1 & - \\
\hline 51.6529 & 88.29 & 110.5 & 237.8 & - & 0.9876 & 0.9993 & 1 & - \\
\hline 62.5000 & 87.09 & 113.5 & 246.2 & - & 0.9997 & 0.9985 & 1 & - \\
\hline 81.6327 & 85.29 & 116.5 & 259.5 & - & 0.9942 & 0.9997 & 1 & - \\
\hline
\end{tabular}

Tablo 6. PBÇK yapısında rezonans enerji seviyeleri ve bu seviyeler denk gelen elektron enerjilerinde geçiş olasılığ $L_{W}=4 \mathrm{~nm}, \alpha=4 \mathrm{~nm}, V_{L}=V_{M}=V_{R}=$ $250 \mathrm{meV}$

\begin{tabular}{|l|l|l|l|l|l|l|l|l|}
$\begin{array}{c}\boldsymbol{\sigma} \\
\text { meV }\end{array} \mathrm{nm}^{2}$ & $\begin{array}{l}E_{\text {res1 }} \\
\text { meV }\end{array}$ & $\begin{array}{l}E_{\text {res2 }} \\
\text { meV }\end{array}$ & $\begin{array}{l}E_{\text {res3 }} \\
\text { meV }\end{array}$ & $\begin{array}{l}E_{\text {res4 }} \\
\text { meV }\end{array}$ & $T_{\text {Eres1 }}$ & $T_{\text {Eres2 }}$ & $T_{\text {Eres3 }}$ & $T_{\text {Eres4 }}$ \\
\hline 10 & 112.3 & 118.3 & 179 & & 0.9208 & 0.6396 & 1 & - \\
\hline 20.4082 & 111.1 & 126.7 & 165.2 & 236.6 & 0.9923 & 0.9986 & 1 & 1 \\
\hline 40.0000 & 99.1 & 124.3 & 127.9 & 219.2 & 0.9983 & 1 & 1 & 1 \\
\hline 51.6529 & 94.29 & 119.5 & - & 233.6 & 0.9987 & 0.998 & - & 1 \\
\hline 62.5000 & 91.29 & 115.9 & - & 245.6 & 1 & 0.9982 & - & 1 \\
\hline 81.6327 & 87.09 & 109.9 & - & - & 0.9998 & 0.9997 & - & - \\
\hline
\end{tabular}

\section{TARTIŞMA VE SONUÇ}

Düşük boyutlu kuantum sistemlerinde rezonans tünelleme karakteristiklerinin ve enerji seviyelerinin dış alanlara (elektrik, manyetik veya lazer) ve yapının geometrik parametrelerine bağlılığının anlaşılması ve kontrol edilebilirliğinin sağlanması teknolojik açıdan oldukça önemlidir. Kuyu genişliği ve bariyer yükseklikleri enerji seviyesinin yerinin belirlenmesinde etkili yapısal parametrelerdir. Çalışmamızda yoğun lazer alanı altında üçlü ters parabolik kuantum bariyer çift kuyu potansiyelinde elektronik iletim ve enerji seviyelerinin bariyer genişlikleri, lazer alanı giydirme parametresine bağlılığı araştırılmıştır. Hesaplama yöntemi olarak denge-dışı Green fonksiyonları kullanılmıştır. Elde edilen sayısal sonuçlar, yüksekfrekanslı yoğun lazer alanının potansiyel profilini oldukça etkilediğini göstermektedir. Ayrıca, tünelleme geçiş katsayısının kuyu genişliğine, bariyerlerin genişlik parametresi ile uygulanan lazer alanının şiddetine oldukça bağlı olduğu görülmüştür. Enerji seviyeleri ile iletim katsayısının, başka bir kontrol mekanizması olmaksızın lazer alan şiddetinin değiştirilmesi ile kontrol edilebildiği görülmektedir. 


\section{KAYNAKLAR}

[1] Tsu, R. ve Esaki, L. (1973). Tunneling in a finite superlattice, Appl. Phys. Lett., 22, 562-564.

[2] Levi, A.F.J. (2006). Applied Quantum Mechanics, Cambridge University Press.

[3] Mizuta, H. ve Tanoue, T. (1995). Physics and Applications of Resonant Tunneling Diodes, Cambridge University Press.

[4] Aktas, S., Kes, H., Boz, F.K. ve Okan, S.E. (2016). Control of a resonant tunneling structure by intense laser fields, Superlattices and Microstruct., 98, 220-227.

[5] Miyamoto, T., Yamaguchi, A. ve Mukai, T. (2016) Terahertz imaging system with resonant tunneling diodes, Jpn. J. Appl. Phys., 55, 032201.

[6] Rong, T., Yang, L.-A., Yang, L. ve Hao, Y. (2018). Theoretical investigation into negative differential resistance characteristics of resonant tunneling diodes based on lattice-matched and polarization-matched AlInN/ GaN heterostructures, J. Appl. Phys., 123, 045702.

[7] Shen, W.P. ve Rustgi, M.L. (1993). Two coupled parabolic wells under an electric field, J. Appl. Phys., 74, 4006-4014.

[8] Ohmukai, M. (2005). Triangular double barrier resonant tunneling, Mater. Sci. Eng. B, 116, 8790.

[9] Wang, H., Xu, H. ve Zhang, Y. (2006). A theoretical study of resonant tunneling characteristics in triangular double-barrier diodes, Phys. Lett. A, 355, 481-488.

[10] Karmakar, R., Biswas, A., Mukherjee, S. ve Deyasi, A. (2011). Calculating Transmission Coefficient of Double Quantum Well Triple Barrier Structure having Parabolic Geometry using Propagation Matrix Method, IJEAT, 1, 3741.

[11] Chang, L.L., Esaki, L. ve Tsu, R. (1974). Resonant tunneling in semiconductor double barriers, Appl. Phys. Lett., 24, 593-595.

[12] Nutku, F. (2014). Quasi-bound levels, transmission and resonant tunneling in heterostructures with double and multi rectangular, trapezoidal, triangular barriers, $J$. Comput. Electron., 13, 456-465.

[13] Bat1, M., Sakiroglu, S., Sokmen, I. (2016). Electron transport in electrically biased inverse parabolic double-barrier structure, Chin. Phys. B, 25, 057307.

[14] Bat1, M. (2018). Resonant tunneling properties of inverted Morse double quantum barrier, Chin. J. Phys., 56, 593-597.

[15] Davies, J.H. (1998). The Physics of Lowdimensional Semiconductors. An Introduction, Cambridge University Press.

[16] Bati, M. (2019). Electronic Transport and Resonant Tunneling Properties of Hyperbolic Pöschl-Teller Double-Barrier Structures, $J$. Comput. Theor. Transp., 48:2, 66-76.
[17] Ferry, D.K., Goodnick, S.M. ve Bird, J. (2009). Transport in Nanostructures, Cambridge University Press, Cambridge.

[18] Martinz, S.D.G. ve Ramos, R.V. (2016). Double quantum well triple barrier structures: analytical and numerical results, Can. J. Phys., 94(11), 1180-1188.

[19] L.D. Macks, S.A. Brown, R.G. Clark, R.P. Starrett, M.A. Reed, M.R., Deshpande, C.J.L. Fernando ve W.R. Frensley, (1996). Resonant tunneling in double-quantum-well triple-barrier heterostructures, Phys. Rev. B, 54 4857-4862.

[20] Peralta, X.G., Allen, S.J., Wanke, M.C., Harff, N.B., Simmons, J.A., Lilly, M.P., Reno, J.L., Burke, P.J. ve Eisentein, J.P. (2002). Terahertz photoconductivity and plasmon modes in doublequantum-well field-effect transistors, Appl. Phys. Lett., 81, 1627-1629.

[21] Wecker, T., Callsen, G., Hoffmann, A., Reuter, D. ve As, D.J. (2018). Correlation of the Carrier Decay Time and Barrier Thickness for Asymmetric Cubic GaN/A10.64Ga0.36N Double Quantum Wells, Phys. Status Solidi (b), 255, 1700373.

[22] Waho, T., Chen, K. ve Yamamoto, M. (1996). A novel multiple-valued logic gate using resonant tunneling devices, IEEE Electron Device Lett., 17, 223-225.

[23] Lee, J., Lee, J. ve Yang, K. (2012). A low-power 40-gb/s 1: 2 demultiplexer ic based on a resonant tunneling diode, IEEE Trans Nanotechnol., 11, 431-434.

[24] Singh, M.M ve Siddiqui, M.J. (2016). Effect of Si-delta doping and barrier lengths on the performance of triple barrier GaAs/AlGaAs resonant tunneling diode, IEEE International Conference on Electron Devices and Solid-State Circuits (EDSSC), Hong Kong, 30-34.

[25] Markelz, A.G. ve Asmar, N.G. (1996). Interband impact ionization by terahertz illumination of InAs heterostructures, Appl. Phys. Lett., 69, 3975.

[26] Lima, F.M.S., Amato, M.A., Nunes, O.A.C., Fonseca, A.L.A., Enders, B.G. ve da Silva, E.F. (2009). Unexpected transition from single to double quantum well potential induced by intense laser fields in a semiconductor quantum well, $J$. Appl. Phys., 105, 123111.

[27] Eseanu, N., Niculescu, E.C. ve Burileanu, L.M. (2009). Simultaneous effects of pressure and laser field on donors in $\mathrm{GaAs} / \mathrm{Ga}_{1-\mathrm{x}} \mathrm{Al}_{\mathrm{x}}$ As quantum wells, Physica E, 41, 1386-1392.

[28] Eseanu, N. (2011). Intense laser field effect on the interband absorption in differently shaped near-surface quantum wells, Phys. Lett. A, 375(6), 1036-1042.

[29] Chakraborty, T., Manaselyan, A., Barseghyan, M. ve Laroze, D. (2018). Controllable continuous evolution of electronic states in a single quantum ring, Phys. Rev. B, 97, 041304. 
[30] Ungan, F., Mora-Ramos, M.E., Barseghyan, M.G., Pérez, L.M. ve Laroze, D. (2019). Intersubband optical properties of a laser-dressed asymmetric triple quantum well nanostructure, Physica E, 114, 113647.

[31] Barseghyan, M.G., Baghramyan, H.M., Kirakosyan, A.A. ve Laroze, D. (2020). The transition from double to single quantum dot induced by $\mathrm{THz}$ laser field, Physica E, 116, 113758.

[32] Salén, P., Basini, M., Bonetti, S., Hebling, J., Krasilnikov, M., Nikitin, A.Y., Shamuilov, G., Tibai, Z., Zhaunerchyk, V. ve Goryashko, V. (2019). Matter manipulation with extreme terahertz light: Progress in the enabling $\mathrm{THz}$ technology, Phys. Rep., 836-837, 1-74.

[33] Niculescu, E.C., Eseanu, N. ve Spandonide, A. (2015). Laser Field Effects on The Interband Transitions in Differently Shaped Quantum Wells, U.P.B. Sci. Bull., Series A, 77.

[34] Yesilgul, U., Al, E.B., Martínez-Orozco, J.C., Restrepo, R.L., Mora-Ramos, M.E., Duque, C.A., Ungan, F. ve Kasapoglu, E. (2016). Linear and nonlinear optical properties in an asymmetric double quantum well under intense laser field: Effects of applied electric and magnetic fields, Opt. Mater., 58, 107-112.
[35] Saczuk, E. ve Kamiński, J. Z. (2003). Resonant tunneling in the presence of intense laser fields, Phys. Stat. Sol. (b), 240, 273-284.

[36] Aktas, S., Bilekkaya, A., Boz, F.K. ve Okan, S.E. (2015). Electron transmission in symmetric and asymmetric double-barrier structures controlled by laser fields, Superlatt. and Microstruct., 85, 266-273.

[37] Datta, S. (2005). Quantum Transport: Atom to Transistor, Cambridge University Press, Cambridge

[38] Bati, M., (2020) The effects of the intense laser field on the resonant tunneling properties of the symmetric triple inverse parabolic barrier double well structure, Physica B, 594, 412314.

[39] Marinescu, M. ve Gavrila, M. (1996). First iteration within the high-frequency Floquet theory of laser-atom interactions, Phys. Rev. A, 53, 25132521.

[40] Boz, F.K., Aktas, S., Bekar, B. ve Okan, S.E. (2012). Laser field-driven potential profiles of double quantum wells, Phys. Lett. A, 376, 590594. 